\title{
Consumo de Drogas, Rede e Apoio Social entre Pacientes Psiquiátricos Ambulatoriais
}

\author{
Clarissa Mendonça Corradi-Webster ${ }^{1}$, Carolina Magro de Santana Braga, Manoel Antônio dos Santos \\ Universidade de São Paulo, Ribeirão Preto-SP, Brasil
}

\section{RESUMO}

O consumo de drogas é elevado entre pessoas com quadros psicopatológicos e pode acarretar diversos prejuízos que se sobrepõem à morbidade psiquiátrica. A rede e o apoio social podem estar associados ao prognóstico desses indivíduos. O objetivo deste estudo foi identificar o uso problemático de drogas em indivíduos em tratamento psiquiátrico e verificar a associação entre o consumo e medidas de rede e do apoio social. A amostra foi composta por 178 participantes. Foram utilizados os instrumentos: Formulário de informações sociodemográficas e clínicas, ASSIST, MOS-SSS e Inventário sobre a Rede Social. Obteve-se que 41,6\% dos participantes faziam uso problemático de drogas, sendo álcool, tabaco e maconha as mais utilizadas. Ter sido alvo de discriminação, não residir com parceiro fixo e não ser praticante de religião foram associados ao consumo problemático de drogas. Destaca-se a importância da avaliação de fatores ambientais na co-ocorrência do uso problemático de drogas e quadros clínicos psiquiátricos.

Palavras-chave: psicopatologia; drogas; apoio social.

\section{ABSTRACT - Drug Use, Networking, and Social Support among Outpatient Psychiatric Patients}

The consumption of drugs is high among people with psychopathological diagnoses and can cause harm that overlaps the psychiatric morbidity. The social network and support are associated with the prognosis of this population. The aim of this study was to identify problematic drug use among individuals undergoing psychiatric treatment and to verify the association between this problematic consumption and their social network and support measures. The sample consisted of 178 participants. An instrument composed of sociodemographic and clinical questions; the ASSIST; MOS-SSS; and the Social Networks Inventory were used for the data collection. It was found that $41.6 \%$ of the participants presented problematic drug use, the most used substances being alcohol, tobacco and marijuana. Having experienced discrimination, not living with a steady partner and not practicing religion were associated with the problematic drug use. The importance of environmental factors in the co-occurrence of problematic drug use and psychiatric diagnoses is highlighted.

Keywords: Psychopathology; Drugs; Social support.

\section{RESUMEN - Uso de Drogas, Red y Apoyo Social entre Pacientes Psiquiátricos Ambulatorios}

El consumo abusivo de las drogas es grande entre las personas con cuadro clínico psicopatológico y puede generar daños que superponen con la morbilidad psiquiátrica. La red y el apoyo social pueden asociarse con el pronóstico de los individuos. El objetivo de este estudio fue identificar el uso problemático de drogas entre los individuos sometidos a tratamiento psiquiátrico y averiguar la relación entre consumo y medidas de la red y el apoyo social. La muestra fue compuesta por 178 participantes. Fueran utilizados los instrumentos: Formulario de Información Sociodemográfica y Clínica, ASSIST, MOS-SSS y el Inventario sobre Red Social. Se encontró que $41,6 \%$ de los participantes eran adictos a las drogas, siendo el alcohol, el tabaco y la marihuana las más utilizadas. Se asoció a la adicción a las drogas haber sufrido discriminación, no residir con pareja estable y no ser practicante religioso. Se resaltó la importancia de los factores ambientales en la co-ocurrencia de consumo problemático de las drogas y cuadros clínicos psiquiátricos. Palabras clave: Psicopatología; Drogas; Apoyo social.

A comorbidade psiquiátrica entre usuários de substâncias psicoativas refere-se à presença simultânea de pelo menos outro transtorno psiquiátrico em um indivíduo diagnosticado com transtorno relacionado ao uso de substâncias (Torrens, Rossi, Martinez-Riera, MartinezSanvisens, \& Bulbena, 2012). O uso problemático de drogas é elevado entre indivíduos que apresentam quadro clínico psiquiátrico (Clark, Power, Fauve, \& Lopes, 2008; Katz et al., 2012). Dados de pesquisa de prevalência nacional realizada nos Estados Unidos indicaram que 33,1\% das pessoas com diagnósticos psiquiátricos fazem uso abusivo ou são dependentes de drogas lícitas ou ilícitas (Substance Abuse and Mental Health Services Administration [SAMHSA], 2004). Estudo brasileiro realizado com

${ }^{1}$ Endereço para correspondência: Departamento de Psicologia da Faculdade de Filosofia, Ciências e Letras de Ribeirão Preto da Universidade de São Paulo. Avenida dos Bandeirantes, 3900, Campus da USP, 14040-901, Ribeirão Preto, SP. E-mail: clarissac@usp.br

Suporte: Fundação de Amparo à Pesquisa do Estado de São Paulo (FAPESP). Processos n² 2011/22739-2 e n 2012/11849-4 
pacientes psiquiátricos ambulatoriais obteve que 33,9\% faziam uso problemático de álcool (Corradi-Webster, Laprega \& Furtado, 2009).

Pacientes com comorbidades representam um contingente significativo de pessoas em tratamento. Estudo conduzido com indivíduos com transtornos psicóticos, seguidos ambulatorialmente ou em internação, encontrou que $43,5 \%$ dos participantes preencheram critérios para, pelo menos uma vez na vida, diagnóstico de transtornos por uso de substâncias psicoativas (Bizzarri et al., 2009). Destes, 22,0\% tinham transtornos pelo uso de álcool e 38,0\% transtornos por uso de outras substâncias (maconha, sedativos, cocaína, alucinógenos, estimulantes e opioides). Foi observado também que a maconha foi a droga ilícita mais consumida, seguida pela cocaína (Bizzarri et al., 2009). As drogas mais utilizadas podem variar de acordo com a cultura e disponibilidade, sendo cocaína e heroína menos frequentes e álcool, tabaco e maconha as mais consumidas (Hauli, Ndetei, Jande, \& Kabangila, 2011).

Os profissionais de saúde relatam encontrar dificuldades em realizar diagnóstico psicológico e psiquiátrico em situações em que há comorbidades entre transtornos por uso de substâncias psicoativas e outros transtornos mentais (Peuker, Rosemberg, Cunha, \& Araújo, 2010). Nesse sentido, a presença de co-ocorrência de transtornos parece ser um fator complicador para a compreensão diagnóstica e a proposição interventiva em saúde.

Independentemente das diferentes prevalências encontradas, a co-ocorrência do uso problemático de drogas e transtornos psiquiátricos está associada a pior prognóstico, quando comparada à ocorrência de apenas um quadro clínico psiquiátrico, além de que os pacientes apresentam maior gravidade do transtorno, tanto do ponto de vista clínico como social (Torrens et al., 2012). Quando há a co-ocorrência, é relatada na literatura científica maior presença de comportamentos violentos (Erkiran et al., 2006), episódios depressivos em maior número e mais severos, maiores taxas de tentativa de suicídio (Blanco et al., 2012), maiores taxas de recaída e internações (Schmidt, Hesse, \& Lykke, 2011), problemas com a Justiça (Mueser \& Gingerinch, 2013), instabilidade de moradia, diminuição da capacidade funcional, infecção por HIV e baixa adesão à medicação (Drake \& Brunette, 1998).

Pesquisa que entrevistou pacientes que passaram por internação psiquiátrica verificou que há associação entre o uso de maconha e de álcool com maior sintomatologia psicopatológica e também com funcionamento psicossocial e ocupacional rebaixado (Bahorik, Newhill, \& Eack, 2013). Destaca-se estudo que analisou a associação da comorbidade de transtornos psicóticos ou de humor com abuso ou dependência de maconha e problemas psicossociais (problemas com o grupo de apoio primário, educacionais, ocupacionais, de habitação, econômicos, com o acesso a serviços de saúde e relacionados à interação com o sistema legal), encontrando associação entre a comorbidade e ter um ou mais dos problemas psicossociais descritos (Compton, Simmons, Weiss, \& West, 2011).

Os efeitos do tratamento também ficam comprometidos no caso da comorbidade psiquiátrica. Estudo que avaliou a eficácia de tratamentos para a dependência do álcool e para depressão concluiu que ambos tendem a ser menos efetivos quando a comorbidade está presente (Hall, Lynskey, \& Teeson, 2001). Diante desses dados, ressalta-se que a associação entre o uso problemático de drogas e transtornos psiquiátricos parece cronificar e agravar os quadros clínicos, trazendo inúmeros prejuízos e exigindo programas terapêuticos e de reabilitação a longo prazo (Di Lorenzo, Galliani, Guicciardi, Landi, \& Ferri, 2014).

A co-ocorrência de diferentes sintomatologias e quadros clínicos psiquiátricos com o uso problemático de drogas tem seu curso e prognóstico altamente influenciada, entre outros aspectos, pelos fatores ambientais, como a presença de rede e apoio sociais (Brown, Bennett, \& Bellack., 2011; Mueser et al., 2000). Estudo com indivíduos em tratamento psiquiátrico ambulatorial, que também faziam uso problemático de drogas, concluiu que menor adesão ao medicamento estava associada com os efeitos colaterais dele, baixa autoeficácia e reduzido apoio social (Magura, Rosenblum, \& Fong, 2012). Intervenções direcionadas a fortalecer a rede social e propiciar oportunidades de apoio social têm obtido melhores resultados. Em estudo realizado em diferentes centros de atendimento, com indivíduos com comorbidade psiquiátrica, foi encontrada associação entre frequentar grupo de ajuda mútua e resultados positivos no que concerne ao uso de drogas (Rosenblum et al., 2014).

Estudo francês com indivíduos que realizavam tratamento para dependência de drogas e apresentavam algum transtorno psiquiátrico, indicou que a rede social em que estão inseridos influencia de forma paradoxal, ora contribuindo para a diminuição do consumo, ora colaborando para seu aumento, já que há evidências do uso de drogas como estratégia facilitadora das relações interpessoais (Acier, Nadeau, \& Landry, 2011). Levantamento realizado com mulheres com duplo diagnóstico psiquiátrico concluiu que o suporte dos amigos para a abstinência de drogas e o apoio para recuperação são preditores de qualidade de vida. Intervenções que incidem sobre o apoio social e a qualidade de vida de mulheres com transtornos por uso de drogas podem melhorar os resultados do tratamento (Brown, Jun, Min, \& Tracy, 2013).

O impacto positivo do apoio social na saúde já é discutido na literatura científica desde a década de 1970. Cobb (1976) descreveu os benefícios do apoio social, sendo este indicado como fenômeno protetivo de diversas patologias orgânicas (como artrite, tuberculose, entre outras) e psíquicas (depressão, alcoolismo e outros 
diagnósticos psiquiátricos). Além de favorecer a prevenção, o apoio social está relacionado à melhor adesão ao tratamento e menor quantidade de medicamentos necessários para a recuperação (Cobb, 1976).

Desde os primeiros apontamentos sobre o tema até o momento atual, a literatura científica é enfática quanto aos efeitos positivos do apoio social e o eventual agravamento dos problemas de saúde, dos tratamentos e do uso dos serviços de saúde decorrente da ausência deste apoio (Canesqui \& Basarglini, 2012). Entretanto, não há consenso a respeito da definição de apoio e rede social e da interseção entre esses dois construtos, sendo, portanto, conceitos complexos, compostos por várias dimensões (Canesqui \& Basarglini, 2012). Uma das primeiras definições acerca do tema foi descrita por Cobb (1976), que caracterizou o apoio social como: "qualquer informação que leve o sujeito a acreditar que ele é cuidado e amado, estimado e valorizado e que pertence a uma rede de comunicação e obrigação mútua” (p. 300).

Nesse âmbito, existe ainda variedade na nomenclatura utilizada, sendo os termos mais encontrados na literatura científica brasileira: "apoio social", "rede social" e "suporte social", "rede social de apoio", "rede de apoio social", "rede de suporte social", "rede de apoio social e afetivo", "rede de relações", "suporte familiar", "suporte psicossocial", "suporte social de apoio", "apoio familiar" e "apoio psicológico” (Leonidas \& Santos, 2013). Há diversidade também entre os aspectos sob os quais o apoio social pode ser analisado, como, por exemplo, o apoio recebido (o que a pessoa relata estar recebendo ou já ter recebido) e o apoio percebido (apoio que a pessoa acredita estar disponível, caso necessitar) (Gonçalves, Pawlowski, Bandeira, \& Piccinini, 2011).

No presente estudo, o conceito de rede e apoio social está integrado, compreendendo como rede o aspecto estrutural de como são percebidas as relações, enquanto o apoio social corresponde ao aspecto funcional. O conceito de rede social utilizado corresponde ao grupo de pessoas com as quais o indivíduo mantém contato ou alguma forma de vínculo social (Bowling, 1997). O apoio social, por sua vez, é definido como o "grau com que relações interpessoais correspondem a funções específicas" (Sherbourne \& Stewart, 1991, p. 706). Sherbourne e Stewart (1991) avaliam ainda cinco dimensões desse apoio: apoio emocional (expressão de afeto positivo e incentivo à expressão dos sentimentos), apoio afetivo (demonstrações de afeto), interação social positiva (disponibilidade de outras pessoas para realizar atividades divertidas e passar momentos agradáveis), apoio de informação (receber aconselhamento, informação ou orientação) e apoio material (disponibilidade de recursos materiais e serviços).

Diante do exposto até o momento, depreende-se que a literatura científica oferece evidências de que o uso problemático de drogas é elevado entre pessoas com quadros clínicos psicopatológicos (Clark et al., 2008;
Katz et al. 2012) e que essa associação pode trazer prejuízos ao funcionamento individual e agravamento dos sintomas (Torrens et al., 2012). A rede e o apoio social também parecem estar associados ao prognóstico de pessoas em tratamento para quadros clínicos psiquiátricos, sendo importantes preditores de saúde (Warren, Stein, \& Grella, 2007). Conhecer melhor como as variáveis relacionadas à rede e ao apoio social se relacionam com o uso problemático de drogas entre pessoas em tratamento psiquiátrico pode auxiliar profissionais que atuam na área a oferecerem um cuidado mais efetivo e amplo a essas pessoas. Assim, o objetivo deste estudo foi identificar o uso problemático de drogas entre indivíduos em tratamento psiquiátrico em um serviço ambulatorial de saúde mental e verificar a associação entre tal consumo e medidas de percepção da rede e do apoio social.

\section{Método}

\section{Delineamento}

Foi realizado um estudo quantitativo, descritivo-exploratório, de corte transversal.

\section{Participantes}

Os participantes eram usuários de uma unidade ambulatorial de saúde mental de um município do interior do estado de São Paulo. Esse serviço é responsável pelos atendimentos em Psiquiatria para pessoas a partir de 18 anos, residentes no distrito central do município.

Foi composta uma amostra clínica, por conveniência, não probabilística. Os critérios de inclusão na pesquisa foram: estar em atendimento no serviço selecionado, frequentando-o no período da coleta de dados; estar em condições de oferecer informações adequadas ao objetivo do estudo. Foram critérios de exclusão: apresentar sinais clínicos de intoxicação por uso de drogas no momento da coleta de dados; ter antecedentes de transtornos mentais orgânicos ou apresentar sintomas psicóticos agudos.

A amostra foi composta por 178 participantes, sendo 131 (73,6\%) mulheres, idade média de 49,6 \pm 13,6 anos; 79 (44,4\%) eram casados ou amasiados; 108 (60,7\%) interromperam sua escolarização no ensino fundamental. As demais características sociodemográficas desse grupo avaliado foram: 109 (61,2\%) não exerciam atividade remunerada; 132 (74,2\%) tinham renda própria; 116 $(65,2 \%)$ não estavam satisfeitos com a situação financeira; 143 (80,3\%) residiam com familiares e 133 (74,7\%) não tinham filhos com idade menor que 18 anos.

\section{Instrumentos}

Formulário de informações sociodemográficas e clínicas. Questionário de autorrelato com questões que visam à identificação de características sociodemográficas e clínicas dos participantes, como sexo, idade, estado civil, escolaridade, emprego, renda própria, satisfação financeira, tempo de tratamento psiquiátrico, uso 
de medicamentos, histórico de internações psiquiátricas, uso de drogas na família, satisfação com a comunidade onde reside, experiências de discriminação, vivência de morar na rua e problemas com a polícia. Após o preenchimento das questões pelos participantes, os entrevistadores registravam nesse formulário o diagnóstico do paciente, obtido no prontuário médico e realizado pelos médicos psiquiatras do serviço seguindo os critérios da Classificação Internacional de Doenças, versão 10 (CID10) (Organização Mundial de Saúde [OMS], 2003).

ASSIST (Alcohol, Smoking and Substance Involvement Screening Test). Desenvolvido pela Organização Mundial de Saúde (OMS, 2003) para o rastreamento do consumo de drogas em diferentes serviços, incluindo clínicas psiquiátricas e serviços de saúde mental. Foi validado no Brasil por Henrique et al. (2004), com índice de consistência interna considerado satisfatório: alfa de Cronbach de 0,80 para álcool; 0,79 para maconha e 0,81 para cocaína. Com esse instrumento, é possível investigar o consumo de dez diferentes classes de drogas (derivados do tabaco, bebidas alcoólicas, maconha, cocaína/crack, anfetamina/êxtase, inalantes, hipnóticos/ sedativos, alucinógenos, opioides, outros). A partir da soma dos escores dos itens relativos ao consumo de cada substância, classifica-se o usuário como usuário de baixo risco, usuário de risco moderado ou usuário de alto risco para a substância avaliada. A partir dos resultados obtidos neste estudo, adicionou-se uma variável nomeada Uso Problemático de Drogas (UPD), a partir da sugestão de Tantirangsee e Assanangkornchai (2015), que utilizaram o ASSIST para investigar o uso de drogas entre pacientes psicóticos. Receberam o escore 1 todos os participantes que foram classificados como usuários de risco moderado ou de alto risco para qualquer uma das drogas, e escore 0 todos os que não faziam consumo de qualquer das drogas rastreadas ou que faziam consumo de baixo risco.

MOS-SSS (Medical Outcomes Study Questions - Social Support Survey). Instrumento do Medical Outcomes Study (MOS), foi desenvolvido para utilização em pacientes com doenças crônicas e caracteriza-se por avaliar o apoio social de maneira multidimensional, sendo autoaplicável. Foi desenvolvido por Sherbourne e Stewart (1991) e validado no Brasil por Mattos (2009), que obteve $\alpha$ de Cronbach variando de 0,91 a 0,97 e estabilidade variando de 0,72 a 0,78 . No Brasil, já foi utilizado em estudos na área da saúde mental (Costa \& Ludermir, 2005). É composto por 19 questões, que abordam cinco dimensões funcionais de apoio social: material (disponibilidade de recursos materiais e serviços), afetivo (demonstrações de afeto), emocional (expressão de afeto positivo e incentivo e compreensão da expressão dos sentimentos), informação (receber aconselhamento, informação ou orientação) e interação social positiva (disponibilidade de outras pessoas para realizar atividades divertidas e passar momentos agradáveis) (Griep, Chor, Faerstein, \& Lopes, 2003). Para avaliar o apoio social global em cada dimensão, foi considerado como nota de corte 33 pontos. Resultado acima desse valor foi interpretado como indicativo de elevado apoio social (Costa \& Ludermir, 2005). Ressalta-se que o apoio social é definido por esses autores como o "grau em que relações interpessoais correspondem a funções específicas" (Sherbourne \& Stewart, 1991, p. 706).

Inventário sobre a Rede Social (Social Network Index), proposto por Berkman e Syme (1979). Esse instrumento avalia a rede social por meio da identificação de características como número de parentes e amigos íntimos, situação conjugal, participação em grupos e instituições. Foi adaptado para a realidade brasileira, sendo que no processo de aferição o instrumento foi avaliado como adequado à população de estudo (Griep et al., 2003). É importante ressaltar que o aporte teórico que subsidia esse instrumento conceitua rede social como "relações sociais que circundam o indivíduo e os grupos de pessoas com quem há contato ou alguma forma de participação social" (Bowling, 1997; Berkman \& Syme, 1979). Além disso, considerando-se o tema de estudo (uso de drogas) e o destaque que a literatura científica dá para a importância dos grupos de autoajuda na composição da rede de apoio de pacientes (Green, Yarborough, Polen, Janoff, \& Yarborough, 2015), julgou-se importante avaliar também a participação dos entrevistados nesses grupos, sendo acrescentada a pergunta: "Nos últimos 12 meses, você participou de algum grupo de autoajuda, como AA (Alcoólicos Anônimos), Al-Anon (grupo de familiares e amigos de alcoólicos), NA (Narcóticos Anônimos), Amor Exigente, AAPSI (Associação de Apoio ao Psicótico) ou outros?”.

\section{Procedimentos}

O estudo foi aprovado pelo Comitê de Ética em Pesquisa da instituição a qual pertencem os pesquisadores (CAAE - 00670512.9.0000.5407). Foram respeitados os critérios éticos preconizados na resolução brasileira.

Os entrevistadores foram estudantes de graduação e pós-graduação em Psicologia, que foram treinados para a coleta e receberam supervisões semanais da última autora deste artigo. Estes se revezavam durante os dias da semana para irem ao serviço e convidarem possíveis voluntários, pacientes que estavam na sala de espera do ambulatório de Psiquiatria, aguardando a consulta médica. Os voluntários que aceitaram colaborar com o estudo eram convidados a se dirigirem a uma sala reservada. Todos os instrumentos utilizados na coleta de dados são de autorrelato. Durante a aplicação, os entrevistadores mantinham-se próximos ao participante a fim de esclarecer dúvidas quanto às questões e ao modo de preenchimento de cada instrumento.

\section{Análise dos Dados}

As variáveis sociodemográficas e clínicas foram categóricas, à exceção da idade e tempo de tratamento em 
saúde mental, sendo realizada análise inicialmente descritiva dos dados. Recorreu-se ao teste do Qui-Quadrado para comparar distribuição dos casos nas variáveis examinadas, a seguir executando-se o procedimento de odds ratio para avaliar a relação entre a chance dos pacientes possuírem a condição clínica de interesse. A comparação entre as médias de agrupamentos dos casos foi realizada mediante a aplicação do teste de Mann-Whitney, adotando-se o intervalo de confiança de 95\%. Para as análises estatísticas, foi utilizado o software Statistical Package for the Social Sciences (SPSS) versão 20.0. Considerou-se a variável "Uso Problemático de Drogas", gerada a partir do ASSIST, como variável dependente. Testou-se a associação entre esta variável e as demais variáveis sistematizadas no estudo.

\section{Resultados}

O uso problemático de drogas foi identificado por meio do instrumento ASSIST. Primeiramente, foram analisados os três padrões de consumo rastreados por esse instrumento, para cada uma das drogas. Na Tabela 1 , nota-se que não foram apresentados os resultados obtidos para as substâncias "Hipnóticos/sedativos", uma vez que esse grupo abrange medicamentos que são prescritos para grande parte dos pacientes psiquiátricos, o que torna difícil avaliar a confiabilidade das respostas. Também se optou por não apresentar os resultados para "Outras drogas", já que houve grande variabilidade de respostas e algumas das substâncias apresentadas não são consideradas culturalmente como drogas, como café e açúcar.

Tabela 1

Distribuição dos Pacientes Psiquiátricos $(n=178)$ de acordo com os Padrões de Consumo de Drogas

\begin{tabular}{|c|c|c|c|c|c|c|}
\hline \multirow{2}{*}{ Substância } & \multicolumn{2}{|c|}{ Baixo risco } & \multicolumn{2}{|c|}{ Risco moderado } & \multicolumn{2}{|c|}{ Alto risco } \\
\hline & Frequência & $\%$ & Frequência & $\%$ & Frequência & $\%$ \\
\hline Tabaco & 120 & 67,4 & 49 & 27,5 & 9 & 0,1 \\
\hline Álcool & 155 & 87,1 & 19 & 10,7 & 4 & 2,2 \\
\hline Maconha & 174 & 97,8 & 4 & 2,2 & - & - \\
\hline Cocaína/crack & 175 & 98,3 & 2 & 1,1 & 1 & 0,6 \\
\hline Anfetaminas & 177 & 99,4 & - & - & 1 & 0,6 \\
\hline Inalantes & 176 & 98,9 & 2 & 1,1 & - & - \\
\hline Alucinógenos & 175 & 98,3 & 3 & 1,7 & - & - \\
\hline Opióides & 177 & 99,4 & 1 & 0,6 & - & - \\
\hline
\end{tabular}

Com base nos dados apresentados na Tabela 1, os pacientes foram estratificados entre aqueles que faziam uso problemático de pelo menos uma droga $(n=74 ; 41,6 \%)$ e os que não faziam uso problemático de drogas $(n=104 ; 58,4 \%)$. Essa variável foi considerada como variável dependente e buscou-se conhecer sua associação com outras variáveis sociodemográficas e clínicas (Tabela 2).

Tabela 2

Descrição das Características Sociodemográficas e Clínicas dos Participantes e Uso Problemático de Drogas

\begin{tabular}{|c|c|c|c|c|c|c|c|c|}
\hline \multirow{2}{*}{ Variável } & & \multicolumn{2}{|c|}{$\begin{array}{c}\text { Uso problemático } \\
\text { de drogas }\end{array}$} & \multirow{2}{*}{$\chi^{2}$} & \multirow{2}{*}{$p$} & \multirow{2}{*}{$\begin{array}{l}\text { Odds } \\
\text { Ratio }\end{array}$} & \multicolumn{2}{|c|}{ IC (95\%) } \\
\hline & & $\begin{array}{l}\text { Não } \\
N(\%) \\
\end{array}$ & $\begin{array}{c}\text { Sim } \\
N(\%)\end{array}$ & & & & Lower & Upper \\
\hline \multirow{2}{*}{ Sexo } & Masc. & $23(48,9)$ & $24(51,1)$ & 2,37 & 0,12 & 0,59 & 0,30 & 1,16 \\
\hline & Fem. & $81(61,8)$ & $50(38,2)$ & & & & & \\
\hline \multirow{2}{*}{ Estado Civil ${ }^{*}$} & Sem C & $49(49,5)$ & $50(50,5)$ & 7,33 & $0,00^{b}$ & 0,43 & 0,23 & 0,79 \\
\hline & Com C & $55(69,6)$ & $24(30,4)$ & & & & & \\
\hline \multirow{2}{*}{ Empregado } & Não & $61(56,0)$ & $48(44,0)$ & 0,70 & 0,40 & 0,77 & 0,41 & 1,42 \\
\hline & Sim & $43(62,3)$ & $26(37,7)$ & & & & & \\
\hline \multirow{2}{*}{ Renda própria } & Não & $30(65,2)$ & $16(34,8)$ & 1,18 & 0,28 & 0,68 & 0,34 & 1,37 \\
\hline & Sim & $74(56,1)$ & $58(43,9)$ & & & & & \\
\hline \multirow{2}{*}{ Prática religiosa ${ }^{a}$} & Não & $28(48,3)$ & $30(51,7)$ & 6,13 & $0,01^{c}$ & 2,32 & 1,18 & 4,55 \\
\hline & Sim & $65(68,4)$ & $30(31,6)$ & & & & & \\
\hline
\end{tabular}


Tabela 2 (continuação)

Descrição das Características Sociodemográficas e Clínicas dos Participantes e Uso Problemático de Drogas

\begin{tabular}{|c|c|c|c|c|c|c|c|c|}
\hline \multirow{2}{*}{ Variável } & & \multicolumn{2}{|c|}{$\begin{array}{c}\text { Uso problemático } \\
\text { de drogas }\end{array}$} & \multirow{2}{*}{$\chi^{2}$} & \multirow{2}{*}{$p$} & \multirow{2}{*}{$\begin{array}{l}\text { Odds } \\
\text { Ratio }\end{array}$} & \multicolumn{2}{|c|}{ IC (95\%) } \\
\hline & & $\begin{array}{l}\text { Não } \\
N(\%) \\
\end{array}$ & $\begin{array}{c}\text { Sim } \\
N(\%) \\
\end{array}$ & & & & Lower & Upper \\
\hline \multirow{2}{*}{ Residência com familiar } & Não & $19(54,3)$ & $16(45,7)$ & 0,31 & 0,58 & 0,81 & 0,38 & 1,70 \\
\hline & Sim & $85(59,4)$ & $58(40,6)$ & & & & & \\
\hline \multirow{2}{*}{ Internação psiquiátrica } & Não & $77(57,5)$ & $57(42,5)$ & 0,21 & 0,65 & 1,18 & 0,59 & 2,36 \\
\hline & $\operatorname{Sim}$ & $27(61,4)$ & $17(38,6)$ & & & & & \\
\hline \multirow{2}{*}{ Familiar com uso de drogas } & Não & $48(56,5)$ & $37(43,5)$ & 0,26 & 0,61 & 1,17 & 0,64 & 2,12 \\
\hline & $\operatorname{Sim}$ & $56(60,2)$ & $37(9,8)$ & & & & & \\
\hline \multirow{2}{*}{ Sofreu violência } & Não & $65(60,7)$ & $42(39,3)$ & 0,59 & 0,44 & 0,79 & 0,43 & 1,45 \\
\hline & Sim & $39(54,9)$ & $32(45,1)$ & & & & & \\
\hline \multirow{2}{*}{ Sofreu discriminação } & Não & $66(71,0)$ & $27(29,0)$ & 12,61 & $0,00^{\mathrm{b}}$ & 0,33 & 0,18 & 0,61 \\
\hline & Sim & $38(44,7)$ & $47(55,3)$ & & & & & \\
\hline
\end{tabular}

Nota. ${ }^{*}$ Sem C=sem companheiro; Com C=com companheiro; a: $n=153 ; b: p<0.001 ; c$ : $p<0,05$

As questões relativas à rede social foram respondidas por 170 participantes. A primeira questão do Inventário sobre a Rede Social refere-se ao número de parentes com quem o paciente se sente a vontade e com quem pode falar sobre quase tudo. A média encontrada foi $2,01 \mathrm{pa}-$ rentes $(D P=2,27)$. A segunda questão refere-se ao número de amigos com quem se sente a vontade. A média encontrada foi 1,39 amigos $(D P=3,02)$. Nestes dois casos, com o uso de teste de Mann-Whitney verificou-se que não há diferenças no número de parentes $(p=0,110)$ e de amigos $(p=0,757)$ entre pacientes que faziam uso problemático de drogas e daqueles que não faziam. Com o teste de qui-quadrado buscou-se conhecer se havia associação entre participação em diferentes grupos sociais e o uso problemático de drogas. A Tabela 3 também apresenta os resultados do teste de qui-quadrado para as cinco dimensões de apoio social avaliadas pelo instrumento MOOS.

Tabela 3

Características da Rede Social dos Participantes, Descrição do Apoio Social em Cinco Diferentes Dimensões e Uso Problemático de Drogas

\begin{tabular}{|c|c|c|c|c|c|}
\hline \multirow{2}{*}{ Rede Social (no último ano) } & & \multicolumn{2}{|c|}{$\begin{array}{c}\text { Uso problemático } \\
\text { de drogas }\end{array}$} & \multirow[b]{2}{*}{$\chi^{2}$} & \multirow[b]{2}{*}{$p$} \\
\hline & & $\begin{array}{l}\text { Não } \\
N(\%)\end{array}$ & $\begin{array}{l}\text { Sim } \\
N(\%)\end{array}$ & & \\
\hline \multirow{2}{*}{$\begin{array}{l}\text { Participação em atividades artísticas } \\
\text { ou esportivas em grupo* }\end{array}$} & Não & $84(60,4)$ & $55(39,6)$ & \multirow[t]{2}{*}{2,42} & \multirow[t]{2}{*}{0,120} \\
\hline & Sim & $14(45,2)$ & $17(54,8)$ & & \\
\hline \multirow{2}{*}{$\begin{array}{l}\text { Participação em reuniões de associações, } \\
\text { sindicatos ou partidos políticos* }\end{array}$} & Não & $87(56,1)$ & $68(43,9)$ & \multirow[t]{2}{*}{1,65} & \multirow[t]{2}{*}{0,198} \\
\hline & Sim & $11(73,3)$ & $04(26,7)$ & & \\
\hline \multirow{2}{*}{ Participação em trabalho voluntário não remunerado* } & Não & $83(56,5)$ & $64(43,5)$ & \multirow[t]{2}{*}{0,63} & \multirow[t]{2}{*}{0,429} \\
\hline & Sim & $15(65,2)$ & $08(34,8)$ & & \\
\hline \multirow{2}{*}{$\begin{array}{l}\text { Participação em igrejas, cultos ou } \\
\text { reuniões religiosas/espirituais* }\end{array}$} & Não & $28(54,9)$ & $23(45,1)$ & \multirow[t]{2}{*}{0,22} & \multirow[t]{2}{*}{0,635} \\
\hline & Sim & $70(58,8)$ & $49(41,2)$ & & \\
\hline \multirow{2}{*}{ Participação em grupos de autoajuda* } & Não & $88(59,5)$ & $60(40,5)$ & \multirow[t]{2}{*}{1,54} & \multirow[t]{2}{*}{0,215} \\
\hline & Sim & $10(45,5)$ & $12(54,5)$ & & \\
\hline \multirow{2}{*}{ Dimensão Afetiva** } & Elevada & $88(56,8)$ & $67(43,2)$ & \multirow[t]{2}{*}{0,01} & \multirow[t]{2}{*}{0,916} \\
\hline & Baixa & $7(58,3)$ & $5(41,7)$ & & \\
\hline \multirow{2}{*}{ Dimensão Emocional** } & Elevada & $82(55,8)$ & $65(44,2)$ & \multirow[t]{2}{*}{0,37} & \multirow[t]{2}{*}{0,542} \\
\hline & Baixa & $12(63,2)$ & $07(36,8)$ & & \\
\hline
\end{tabular}


Tabela 3 (continuação)

Características da Rede Social dos Participantes, Descrição do Apoio Social em Cinco Diferentes Dimensões e Uso Problemático de Drogas

\begin{tabular}{|c|c|c|c|c|c|}
\hline \multirow{2}{*}{ Rede Social (no último ano) } & & \multicolumn{2}{|c|}{$\begin{array}{l}\text { Uso problemático } \\
\text { de drogas }\end{array}$} & \multirow[b]{2}{*}{$\chi^{2}$} & \multirow[b]{2}{*}{$p$} \\
\hline & & $\begin{array}{l}\text { Não } \\
N(\%)\end{array}$ & $\begin{array}{c}\text { Sim } \\
N(\%)\end{array}$ & & \\
\hline \multirow{2}{*}{ Dimensão de Informação** } & Elevada & $86(57,0)$ & $65(43,0)$ & 0,00 & 0,957 \\
\hline & Baixa & $9(56,3)$ & $7(43,8)$ & & \\
\hline \multirow{2}{*}{ Dimensão de Interação Positiva** } & Elevada & $85(57,0)$ & $64(43,0)$ & 0,02 & 0,904 \\
\hline & Baixa & $10(55,6)$ & $8(44,4)$ & & \\
\hline \multirow{2}{*}{ Dimensão Material** } & Elevada & $83(57,6)$ & $61(42,4)$ & 0,24 & 0,623 \\
\hline & Baixa & $12(52,2)$ & $11(47,8)$ & & \\
\hline
\end{tabular}

Nota: ${ }^{*} n=170 ;{ }^{* *} n=167$

Destaca-se que o escore médio para o apoio social global foi 65,9 $(D P=19,0)$. Não foram encontradas diferenças estatisticamente significativas entre as médias de apoio social global dos pacientes que faziam uso problemático de drogas e daqueles que não faziam $(t=0,830$; $p=0,408)$.

\section{Discussão}

Este estudo teve como objetivo identificar o uso problemático de drogas entre indivíduos em tratamento psiquiátrico em um serviço ambulatorial de saúde mental e verificar a associação entre esse consumo e medidas de percepção da rede e do apoio social. Quanto à identificação do uso problemático de drogas, corroborando os dados da literatura científica sobre comorbidade psiquiátrica, as substâncias mais utilizadas foram: álcool, tabaco e maconha (Bizzarri et al., 2009; Hauli et al., 2011). Levantamento nacional realizado com a população geral também encontrou que a maconha foi a droga ilícita mais utilizada (Centro Brasileiro de Informação sobre Drogas Psicotrópicas [CEBRID], 2006).

Quando investigada a provável dependência (uso de alto risco), destaca-se que as prevalências obtidas neste estudo são menores do que as encontradas na população geral em dois levantamentos nacionais populacionais (CEBRID, 2006; Instituto Nacional de Políticas Públicas do Álcool e Outras Drogas [INPAD], 2012). Ao serem comparados com as prevalências descritas em estudos internacionais com pacientes psiquiátricos, observou-se que os dados obtidos neste estudo em relação ao abuso e dependência (moderado e alto risco) estão aquém do esperado, à exceção do uso de tabaco (Hauli et al., 2011; Katz, Durst, Shufman, BarHamburger, \& Grunhaus, 2008). Essa discrepância em relação aos resultados de estudos internacionais pode estar relacionada a diversos fatores, incluindo o ambiente social. Em países desenvolvidos, as políticas públicas para atenção a indivíduos com transtornos psiquiátricos incluem auxílio à moradia, emprego supervisionado e tratamento integral, incluindo visitas domiciliares e obtenção de medicação, estimulando-se a autonomia do paciente. No Brasil, a ausência desse tipo de atenção integral ao indivíduo com transtorno psiquiátrico leva-o a maior dependência da família e, consequentemente, o maior controle que esta exerce sobre a liberdade do indivíduo e sobre o consumo de drogas (Menezes \& Ratto, 2000).

Observou-se também que a percepção do apoio social foi elevada nessa amostra. O contato, em longo prazo, com o serviço de saúde pode auxiliar na percepção de se ter algum apoio, em virtude do vínculo estabelecido com os profissionais e usuários do equipamento de saúde mental. Além disso, o fato de ser uma amostra clínica permite hipotetizar que pessoas com apoio social muito incipiente provavelmente teriam mais dificuldades para manter tratamento por tantos anos, já que o apoio é necessário para sustentar a motivação para a busca de ajuda especializada e também para dar continuidade ao tratamento (Brown et al., 2011).

Nota-se que a rede social percebida foi constituída por poucas pessoas e caracterizada por baixa participação em grupos, o que leva a hipotetizar que o apoio percebido como elevado parece ser fornecido por meio de poucos relacionamentos, podendo evidenciar uma sobrecarga para quem o oferece. Essa hipótese é consistente com dados de estudo brasileiro realizado com familiares de usuários de serviços de saúde mental, que indicou que a maior parte dos cuidadores apresenta elevada sobrecarga objetiva e subjetiva (Barroso, Bandeira, \& Nascimento, 2007). Não foi encontrada associação entre as medidas de percepção da rede e do apoio social e o consumo de drogas recreativas, o que pode estar relacionado a especificidades do grupo avaliado. Neste estudo, o consumo de drogas esteve concentrado em 
uso de baixo risco, o que pode descaracterizar esses indivíduos como necessitando de rede e apoio social para lidar com este nível de consumo.

Por outro lado, algumas características sociais estiveram relacionadas ao consumo de drogas. Encontrouse associação entre já ter sido discriminado e o uso problemático de pelo menos uma das drogas investigadas. Oliver-Quetglas, Torres, March, Socias e Esteva (2013) encontraram associação entre ter sofrido alguma forma de discriminação e o diagnóstico de depressão entre usuários de serviços de saúde. Além disso, sentir-se discriminado ou estigmatizado é muito comum entre as pessoas com diagnósticos psiquiátricos. A literatura científica postula que isso em parte se relaciona às dificuldades que o indivíduo tem em lidar com suas obrigações e compromissos, como manter um trabalho, por exemplo. É comum que pessoas com transtornos mentais tenham maiores taxas de absenteísmo, maior utilização de licença por doença prolongada e que se aposentem mais cedo (Laberon, 2014). Essa situação pode gerar mais estresse na vida do indivíduo, que já é vulnerável por seus sintomas psicopatológicos, aumentando assim o risco de busca por alívio dessas emoções disfóricas por meio do uso de drogas.

Verificou-se também que não residir com um parceiro fixo, sendo ou não casado, foi um fator de risco para o uso problemático de drogas nessa amostra. Esse dado corrobora conclusões de vários estudos na área (Gilmour, 2014; Weich \& Pienaar, 2009), que associam o estado civil (sem companheiro) a maior taxa de recaída entre pessoas com comorbidades psiquiátricas (Menezes \& Ratto, 2004). Estudo canadense também destacou que pessoas com um parceiro fixo eram mais propensas a ter saúde mental mais equilibrada do que aquelas que eram viúvas, separadas, divorciadas ou solteiras (Gilmour, 2014). Residir com um parceiro fixo parece proteger os indivíduos do consumo problemático de drogas, uma vez que o parceiro pode ajudar a lidar com situações de estresse, sem que o sujeito tenha que necessariamente recorrer ao uso de drogas. Além disso, no Brasil muitos pacientes psiquiátricos têm sua autonomia tutelada por membros da família, que cuidam do dinheiro e controlam o uso de drogas (Dunn, 2014).

A ausência de prática religiosa também apareceu como um fator de risco para o uso problemático de drogas. Vale ressaltar que a religião fornece crenças e explicações que ajudam o indivíduo a lidar com situações de estresse, estando por isso associada à saúde mental positiva (Gilmour, 2014). Além disso, quando as pessoas praticam uma religião podem receber apoio do grupo de adeptos de sua crença religiosa, o que auxilia em situações cotidianas e também exerce controle comportamental normatizador.
Um dos limites deste estudo refere-se ao uso de informações sobre o uso de drogas obtidas por meio de resposta do paciente, não sendo consultadas outras fontes nem realizados testes biológicos para verificar a exatidão dos relatos. Houve o cuidado de fornecer aos indivíduos privacidade e conforto, no entanto, esse é um assunto delicado para ser abordado em um primeiro contato e pode, assim, ter havido sub-relato. Outra limitação foi a opção pela amostra de conveniência, não randomizada. Apesar desse tipo de amostragem ser recomendada para verificar se existe o problema em um determinado local e ser considerada como uma técnica útil de se obter informações preliminares a respeito de um aspecto particular da saúde de um grupo (Pereira, 1995), os dados não podem ser usados para prever como o evento se dará na população geral.

\section{Considerações Finais}

A prevalência de uso problemático de drogas encontrada neste estudo é menor do que as descritas em pesquisas realizadas com a população geral e inferior aos achados internacionais. Essa discrepância pode estar relacionada a características socioculturais da amostra e ao fato dos pacientes avaliados estarem já em contato com um serviço de saúde mental, sendo regularmente acompanhados. Isso pode indicar a importância do contato regular com serviços de saúde como um fator de proteção e espaço no qual se pode encontrar ajuda.

Não foi encontrada associação entre medidas de rede e de apoio social e o uso problemático de drogas, embora variáveis sociais tenham sido identificadas como fatores de risco para o uso problemático. Ter sido discriminado, não residir com parceiro fixo e não ter uma prática religiosa foram associados ao consumo problemático de pelo menos uma das drogas avaliadas. Essas associações sugerem a importância de questões sociais e econômicas no consumo de substâncias psicoativas. Os profissionais de saúde devem levar em consideração essas variáveis no planejamento do tratamento para o uso de drogas. Estratégias de enfrentamento baseadas no resolutividade da discriminação, aperfeiçoamento de habilidades interpessoais e afiliação religiosa devem ser encorajadas.

O presente estudo aponta para a importância de fatores ambientais no uso problemático de drogas, reforçando a necessidade dos profissionais de saúde ampliarem o olhar para o contexto sociocultural do usuário em tratamento. Ressalta-se a necessidade de novos estudos que investiguem mais profundamente as variáveis que se mostraram associadas ao uso problemático de drogas nessa amostra, a fim de conhecer possíveis motivos dessa associação. 


\section{Referências}

Acier, D., Nadeau, L., \& Landry, M. (2011). L'influence du réseau social sur La consommation pour dês patients toxicomanes présentant une comorbidité psychiatric. L'encéphale, 37(4), 249-256. doi : 10.1016/j.encep.2010.08.005

Bahorik, A. L., Newhill, C. E., \& Eack, S. M. (2013). Characterizing the longitudinal patterns of substance use among individuals diagnosed with serious mental illness after psychiatric hospitalization. Adicction, 108(7), 1259-1269. doi: 10.1111/add.12153

Barroso, S. M., Bandeira, M., \& Nascimento, E. (2007). Sobrecarga de familiares de pacientes psiquiátricos atendidos na rede pública. Revista de Psiquiatria Clínica, 34(6), 270-277. doi:10.1590/S0101-60832007000600003

Berkman, L. F., \& Syme, S. L. (1979). Social networks, host resistance, and mortality: A nine-year follow-up study of Alameda County residents. American Journal of Epidemiology, 109(2), 186-204. doi:10.1093/oxfordjournals.aje.a112674

Bizzarri, J. V., Rucci, P., Sbrana, A., Miniati, M., Raimondi, F., ... Ravani, L. (2009). Substance use in severe mental illness: Self-medication and vulnerability factors. Psychiatry Research, 165(1-2), 88-95. doi: 10.1016/j.psychres.2007.10.009

Blanco, C., Alegría, A. A., Liu. S. M., Secades-Villa, R., Sugaya, L., Davies, C., \& Nunes, E. V. (2012). Differences among major depressive disorder with and without co-occurring substance use disorders and substance-induced depressive disorder: Results from the National Epidemiologic Survey on Alcohol and Related Conditions. Journal of Clinical Psychiatry, 73(6), 865-873. doi: 10.4088/JCP.10m06673.

Bowling, A. (1997). Measuring social networks and social support. Em A. Bowling (Ed.), Measuring health: A review of quality of life measurements scales (pp. 91-109). Baltimore: Open University Press.

Brown, C.H., Bennett, M. E., Li, L., \& Bellack, A. S. (2011). Predictors of initiation and engagement in substance abuse treatment among individuals with co-occurring serious mental illness and substance use disorders. Addictive Behaviors, 36(5), 439-447. doi: 10.1016/j. addbeh.2010.12.001

Brown, S., Jun, M. K., Min, M. O., \& Tracy, E. M. (2013) Impact of dual disorders, trauma, and social support on quality of life among women in treatment for substance dependence. Journal Of Dual Diagnosis, 9(1), 61-71. doi: 10.1080/15504263.2012.750147

Canesqui, A. M., \& Basarglini, R. A. (2012). Apoio social e saúde: Pontos de vista das ciências sociais e humanas. Ciência E Saúde Coletiva, 17(5), 1103-1114. doi: 10.1590/S1413-81232012000500002

Centro Brasileiro de Informação sobre Drogas Psicotrópicas, [CEBRID] (2006). II Levantamento domiciliar sobre o uso de drogas psicotrópicas no Brasil: Estudo envolvendo as 108 maiores cidades do país: 2005. São Paulo: CEBRID - Centro Brasileiro de Informação sobre Drogas Psicotrópicas: UNIFESP. Recuperado de https://www.cebrid.com.br/wp-content/uploads/2014/10/II-Levantamento-Domiciliarsobre-o-Uso-de-Drogas-Psicotr\%C3\%B3picas-no-Brasil.pdf

Clark, H. E., Power, A. K., Fauve, C. E. L., \& Lopez, E. I. (2008). Policy and practice implications of epidemiological surveys on co-occurring mental and substance use disorders. Journal of Substance Abuse Treatment 34(1), 3-13. doi: 10.1016/j.jsat.2006.12.032

Cobb, S. (1976). Social support as a moderator of life stress. Psychosomatic Medicine, 38(5), 300-314. doi: 10.1097/00006842-197609000-00003

Compton, M. T., Chantelle, M., Simmons, C. M., Weiss, P. S., \& West, J. C. (2011). Axis IV psychosocial problems among patients with psychotic or mood disorders with a cannabis use disorder comorbidity. The American Journal on Addictions, 20(6), 563-567. doi: 10.1111/j.1521-0391.2011.00184.x

Corradi-Webster, C. M., Laprega, M. R., \& Furtado, E. F. (2009). Residentes em psiquiatria têm documentado problemas relacionados ao álcool em pacientes ambulatoriais? Revista de Psiquiatria do Rio Grande do Sul, 31(3), 187-191. doi: 10.1590/S0101-81082009000300008

Costa, A., \& Ludermir, A. (2005). Transtornos mentais comuns e apoio social: Estudo em comunidade rural da Zona da Mata de Pernambuco, Brasil. Cadernos de Saúde Pública, 21(1), 73-79. doi: 79. 10.1590/S0102-311X2005000100009

Di Lorenzo, R., Galliani, A., Guicciardi, A., Landi, G., \& Ferri, P. (2014). A retrospective analysis focusing on a group of patients with dual diagnosis treated by both mental health and substance use services. Neuropsychiatric Disease and Treatment, 10, 1479-1488. doi: 10.2147/ NDT.S65896.

Drake, R. E., \& Brunette, M. F. (1998). Complications of severe mental illness related to alcohol and other drug use disorders. Em M. Galanter (Ed.), Recent developments in alcoholism: Consequences of alcoholism (pp. 285-299). New York, NY: Plenum.

Dunn, M. S. (2014). Association between physical activity and substance use behaviors among high school students participating in the 2009 Youth Risk Behavior Survey. Psychological Reports, 114(3), 675-685. doi: 10.2466/18.06.PR0.114k28w7

Erkiran, M., Özünalan, H., Evren, C., Aytaçlar, S., Kirisci, L., \& Tarter, R. (2006). Substance abuse amplifies the risk of violence in schizophrenia spectrum disorder. Addictive Behaviors, 31(10), 1797-1805. doi:10.1016/j.addbeh.2005.12.024

Gilmour, H. (2014). Positive mental health and mental illness. Health Reports, 25(9), 3-9.

Gonçalves, T. R., Pawlowski, J., Bandeira, D. R., \& Piccinini, C. A. (2011). Avaliação de apoio social em estudos brasileiros: Aspectos conceituais e instrumentos. Ciência \& Saúde Coletiva, 16(3), 1755-1769. doi: 10.1590/S1413-81232011000300012

Green C. A., Yarborough, M. T., Polen, M. R., Janoff, S. L., \& Yarborough, B. J. (2015). Dual recovery among people with serious mental illnesses and substance problems: A qualitative analysis. Journal of Dual Diagnosis, 11(1), 33-41. doi: 10.1080/15504263.2014.975004

Griep, R. H., Chor, D., Faerstein, E., \& Lopes, C. (2003). Apoio social: Confiabilidade teste-reteste de escala no estudo pró-saúde. Cadernos de Saúde Pública, 19(2), 625-634. doi: 10.1590/S0102-311X2003000200029

Hall, W., Lynskey, M., \& Teesson, M. (2001). What is comorbidity and why does it matter? Em M. Teesson \& L. Burns, (Eds.), National comorbidity project. Canberra: Commonwealth Department of Health and Aged Care (pg.11-7). doi:10.1080/ 17523280701747289

Hauli, K. A., Ndetei, D. M., Jande M. B., \& Kabangila, R. (2011). The prevalence of substance use among psychiatric patients: The case study of Bugando Medical Centre, Mwanza (Northern Tanzania). Substance Abuse, 32(4), 238-241. doi: 10.1080/08897077.2011.599253

Henrique, I. F., De Micheli, D., Lacerda, R. B., Lacerda, L. A., Oliveira, M. L., \& Formigoni, S. (2004). Validação da versão brasileira do teste de triagem do envolvimento com álcool, cigarro e outras substâncias (ASSIST). Revista da Associação Médica Brasileira, 50(2), $199-206$. doi: 10.1590/S0104-42302004000200039

Instituto Nacional de Políticas Públicas do Álcool e Outras Drogas [INPAD] (2012). Levantamento Nacional de Álcool e Drogas. Recuperado de http://inpad.org.br/lenad/.

Katz, G., Durst, R., Shufman, E., Bar-Hamburger, R., \& Grunhaus, L. (2008). Substance abuse in hospitalized psychiatric patients. The Israel Medical Association Journal, 10(10), 672-675. Recuperado de https://pdfs.semanticscholar.org/c1d3/157fd91f0b5132dcff936f19e7 6a7e13dd14.pdf 
Katz, G., Grunhaus, L., Deeb, S., Shufman, E., Bar-Hamburger, R., \& Durst, R. (2012). A comparative study of Arab and Jewish patients admitted for psychiatric hospitalization in Jerusalem: The demographic, psychopathologic aspects, and the drug abuse comorbidity. Comprehensive Psychiatry, 53(6), 850-853. doi: 10.1016/j.comppsych.2011.11.005

Laberon S. (2014). Psychological barriers to professional inclusion of people with mental disabilities. Encephale, 40(S2), 103-114. doi: 10.1016/j.encep.2014.04.007

Leonidas, C., \& Santos, M. A. (2013). Redes sociais significativas de mulheres com transtornos alimentares. Psicologia: Reflexão e Crítica, 26(3), 561-571. doi: 10.1590/S0102-79722013000300016

Magura, S., Rosenblum, A., \& Fong, C. (2012). Factors associated with medication adherence among psychiatric outpatients at substance abuse risk. Open Addiction Journal, 4(1), 58-64. doi:10.2174/1874941001104010058.

Mattos, A. (2009). Validade dimensional da escala de apoio social do Medical Outcomes Study adaptada para o português no estudo Pró-Saúde (Dissertação de mestrado). Escola Nacional de Saúde Pública Sérgio Arouca, Rio de Janeiro, RJ. Recuperado de http://www.dominiopublico.gov. br/pesquisa/DetalheObraForm.do?select action $=\&$ co_obra $=165398$

Menezes, P. R., \& Ratto, L. R. (2004). Prevalence of substance misuse among individuals with severe mental illness in São Paulo. Social Psychiatry and Psychiatriac Epidemiology, 34(3), 212-217. doi: 10.1590/S0034-89102006005000039

Mueser, K. T., \& Gingerich, S. (2013). Treatment of co-occurring psychotic and substance. Social Work in Public Health, 28(3-4), 424-439. doi: 10.1080/19371918.2013.774676

Mueser, K. T., Yarnold, P. R., Rosenberg, S. D., Swett, Jr. C., Miles, K. M., \& Hill, D. (2000). Substance use disorder in hospitalized severely mentally ill psychiatric patients: Prevalence, correlates, and subgroups. Schizophrenia Bulletin, 26(1), 179-192.

Oliver-Quetglas, A., Torres, E., March, S., Socias, I. M., \& Esteva, M. (2013). Risk factors of depressive syndrome in young adults. Actas Españolas de Psiquiatría, 41(2), 84-96. Recuperado de https://www.actaspsiquiatria.es/repositorio/15/82/ENG/15-82-ENG-84-96-734131.pdf

Organização Mundial de Saúde [OMS] (2003). Classificação estatística internacional de doenças e problemas relacionados à saúde - CID-10. (9a ed.). São Paulo: Edusp.

Pereira, M. G. (1995). Epidemiologia: teoria e prática. Rio de Janeiro: Guanabara Koogan.

Peuker, A. C., Rosemberg, R., Cunha, S. M., \& Araujo, L. B. (2010). Fatores associados ao abuso de drogas em uma população clínica. Paidéia (Ribeirão Preto), 20(46), 165-173. doi: 10.1590/S0103-863X2010000200004

Rosenblum, A., Matusow, H., Fong, C., Vogel, H., Uttaro, T., Moore, T. L., \& Magura, S. (2014). Efficacy of dual focus mutual aid for persons with mental illness and substance misuse. Drug and Alcohol Dependence, 135, 78-87. doi: 10.1016/j.drugalcdep.2013.11.012

Schmidt, L. M., Hesse, M., \& Lykke, J. (2011) The impact of substance use disorders on the course of schizophrenia - A 15-year follow-up study: Dual diagnosis over 15 years. Schizophrenia Research 130(1-3), 228-233. doi: 10.1016/j.schres.2011.04.011

Sherbourne, C. D., \& Stewart, A. L. (1991). The MOS social support survey. Social Science and Medicine, 32(6), 705-714. doi: 10.1016/02779536(91)90150-B

Substance Abuse and Mental Health Services Administration, Office of Applied Studies [SAMHSA] (2004). National Survey on Drug Use and Health. Recuperado de http://www.oas.samhsa.gov/NSDUH/2k4nsduh/2k4tabs/Sect6peTabs1 to81.htm\#tab6.10b

Tantirangsee, N., \& Assanangkornchai, S. (2015). Prevalence, patterns, associated factors and severity of substance use among psychotic patients in southern Thailand. Asian Journal of Psychiatry, 13, 30-37. doi: 10.1016/j.ajp.2014.11.006

Torrens, M., Rossi, P. C., Martinez-Riera, R., Martinez-Sanvisens, D., \& Bulbena, A. (2012). Psychiatric co-morbidity and substance use disorders: Treatment in parallel systems or in one integrated system? Substance Use \& Misuse, 47(8-9), 1005-1014. doi: 10.3109/10826084.2012.663296

Warren, J. I., Stein, J. A., \& Grella, C. E. (2007). Role of social support and self-efficacy in treatment outcomes among clients with cooccurring disorders. Drug Alcohol Depend, 89(2-3), 267-274. doi: 10.1016/j.drugalcdep.2007.01.009

Weich, L., \& Pienaar, W. (2009). Occurrence of comorbid substance use disorders among acute psychiatric inpatients at Stikland Hospital in the Western Cape, South Africa. African Journal of Psychiatry, 12(3), 213-217. doi: 10.4314/ajpsy.v12i3.48496

recebido em abril de 2019 aprovado em novembro de 2019

\section{Sobre os autores}

Clarissa Mendonça Corradi-Webster é psicóloga (USP), doutora em Psicologia pela Universidade de São Paulo e, atualmente, é professora doutora do Departamento de Psicologia da Faculdade de Filosofia, Ciências e Letras de Ribeirão Preto da Universidade de São Paulo.

Carolina Magro de Santana Braga é psicóloga (USP) e especialista em Neuropsicologia. Atualmente é membro do Laboratório de Ensino e Pesquisa em Psicopatologia, Drogas e Sociedade (LePsis) da Universidade de São Paulo.

Manoel Antônio dos Santos é psicólogo (USP), doutor em Psicologia pela Universidade de São Paulo e, atualmente, é professor titular do Departamento de Psicologia da Faculdade de Filosofia, Ciências e Letras de Ribeirão Preto da Universidade de São Paulo. 